\title{
Infiltrative Intraocular Conjunctival Squamous Cell Carcinoma after Local Resection and Brachytherapy: Clinical and Pathological Findings
}

\author{
Magnolia Trinidad Cano-Suárez ${ }^{a, d} \quad$ María Antonia Saornil-Álvarez ${ }^{a, d}$ \\ Ciro García-Álvarez ${ }^{a, d}$ Francisco López-Larab, d Jesús María Frutos-Barajab, d \\ Elena García-Lagartoc \\ Departments of a Ophthalmology, ${ }^{b}$ Radiotherapy, and ${ }^{\mathrm{C} P a t h o l o g y}$, and d Ocular Oncology Unit, Hospital Clínico \\ Universitario de Valladolid, Valladolid, Spain
}

\section{Established Facts}

- Adjuvant brachytherapy in CSCC can lead to corneal perforation as secondary effect.

\section{Novel Insights}

- CSCC recurrence as intraocular diffuse invasion masquerading as anterior uveitis.

- Clinicopathologic correlation of radiation effects on the ocular tissues after adjuvant brachytherapy for CSCC.

\section{Keywords}

Conjunctival squamous cell carcinoma $\cdot$ Intraocular invasion · Brachytherapy · Radiation · Side effects

\section{Abstract \\ A pseudophakic 70-year-old man presented to the clinic with a slow-growing conjunctival mass in the left eye. He was diagnosed with a conjunctival exophytic lesion suspicious of invasive conjunctival squamous cell carcinoma (CSCC). Exci- sional biopsy showed a well-differentiated CSCC with posi- tive margins and the patient underwent adjuvant brachy- therapy. Six weeks later, examination of the anterior seg-}

ment revealed cells in the anterior chamber and a neurotrophic corneal ulcer with corneal perforation. Considering the high suspicion of intraocular invasion of CSCC, the left eye was enucleated. Histopathologic findings showed scarring of the ciliary body with fibrosis temporally. Nasally, the sclera showed a lobular infiltration of well-differentiated squamous carcinoma. The central cornea exhibited a large ulcer with perforation and infiltrating squamous cells adhering to the posterior surface. To our knowledge, this is the first documented case of an intraocular diffuse recurrence of CSCC after resection and adjuvant brachytherapy, with clinicopathologic correlation of radiation effects on the ocular tissues.

\section{KARGER}

E-Mail karger@karger.com

www.karger.com/oop (c) 2017 S. Karger AG, Basel Magnolia Trinidad Cano-Suárez

Department of Ophthalmology

Hospital Clínico Universitario de Valladolid

St. Ramón y Cajal 3, ES-47005 Valladolid (Spain)

E-Mail magnoliacs@yahoo.com 
Fig. 1. Clinical findings are presented. a A conjunctival nonpigmented vascularized exophytic papillary lesion in the interpalpebral temporal area of the left eye attached to the sclera is presented. b Immunohistochemical study showing a welldifferentiated squamous cell carcinoma positive for CKAE1-AE3 2×. c Slit-lamp examination revealing signs of anterior uveitis and corneal edema, cells in the anterior chamber (hypopyon) and a neurotrophic corneal ulcer. d Magnetic resonance imaging showing an intraocular mass involving the nasal area without signs of orbital involvement.
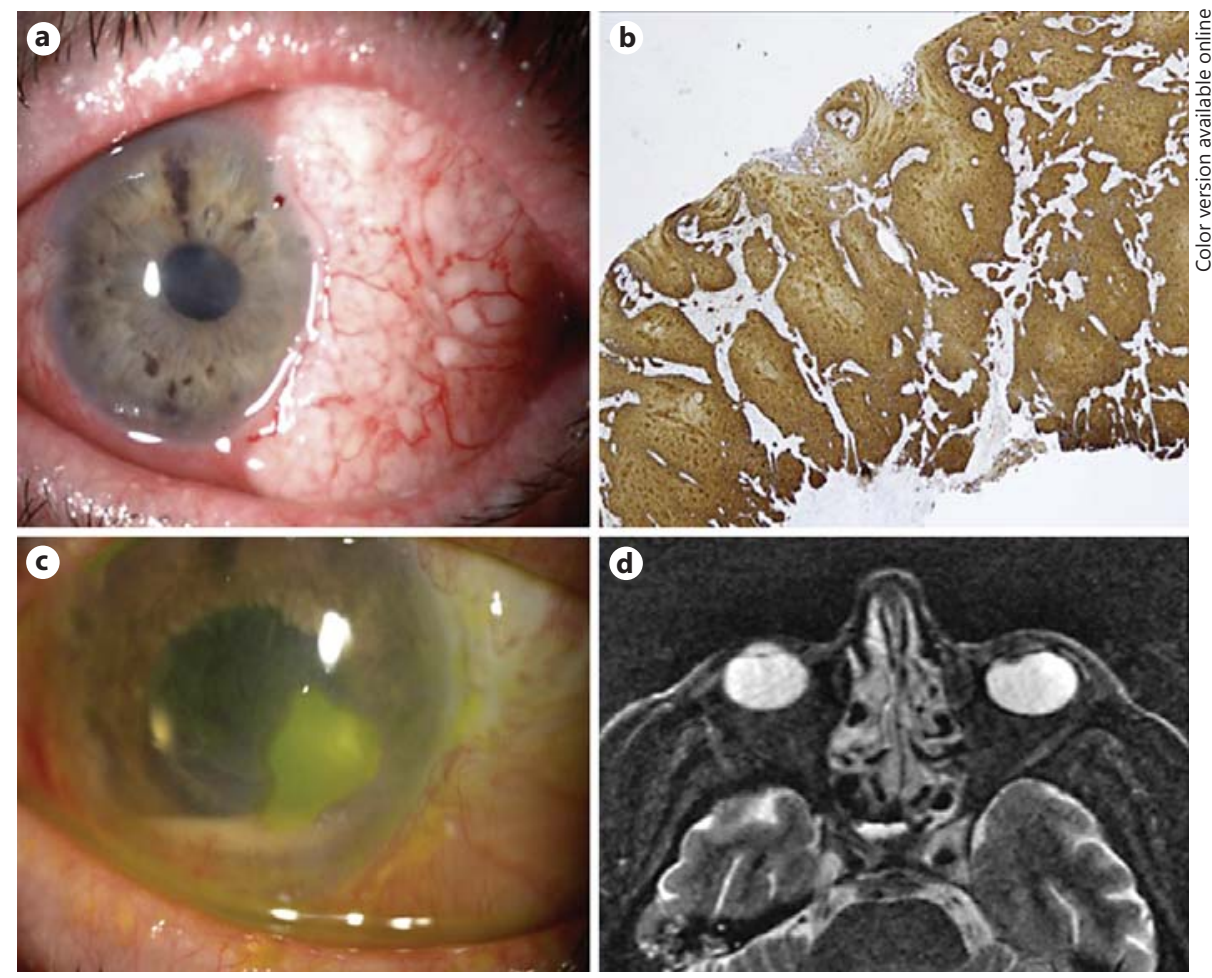

\section{Introduction}

Epithelial conjunctival tumors are named today as ocular surface squamous neoplasia (OSSN). They are considered "in situ" when restricted within the epithelium and "invasive" when infiltrating beyond the confines of the basal membrane as in conjunctival squamous cell carcinoma (CSCC). Invasive CSCC has a low frequency as most of the epithelial lesions are confined to the epithelium $[1,2]$.

In the United States, the estimated incidence of OSSN is 8.4 cases per million. This low-grade malignancy most commonly presents as a slow-growing exophytic mass in the interpalpebral fissure, frequently involving the limbus and is often superficially invasive. It has a relatively benign clinical course and successful outcomes are achieved with excisional biopsy with or without adjunctive therapy. Neglected cases (misdiagnosis or loss to follow-up) or cases associated with immunodeficiency have been linked to more rapid growth and greater tendency to recur, in addition to ocular and orbital invasion. Metastases are extremely infrequent. Intraocular invasion is rare after primary treatment and may be prevented by adjuvant brachytherapy after resection $[1,3]$.

Conjunctival Carcinoma Recurrence with Intraocular Invasion

\section{Case Report}

Our patient is a 70-year-old male, presenting to the clinic with a 1-year history of a slow-growing conjunctival mass. Ocular examination was unremarkable (pseudophakic in both eyes) with the exception of an interpalpebral lesion affecting the temporal bulbar conjunctiva of the left eye (Fig. 1a). The lesion was vascularized, nonpigmented and exophytic/papillary in nature. Ultrasound biomicroscopy showed no intraocular invasion, and a size of $17 \times 15$ $\times 3.4 \mathrm{~mm}$. Considering the high suspicion of invasive squamous conjunctival carcinoma, the patient underwent an excisional biopsy, with a plan for posterior adjuvant therapy. Head and neck computed tomography scan showed no locoregional extension to the lymph nodes. Pathologic study revealed a well-differentiated squamous cell carcinoma with infiltrating lobules, nest and cords with positive deep and lateral margins (Fig. 1b). The patient refused topical adjuvant treatment with mitomycin $\mathrm{C}$ drops due to his social conditions (living alone in a rural setting, unable to keep the adequate administration of drops). One month later, brachytherapy with $\mathrm{Ru} 106$ (20-mm diameter plaque) was used to treat the local intrascleral spread and to prevent recurrences and/or further invasion. Six weeks later, the patient presented with signs of anterior uveitis and corneal edema, which were diagnosed as inflammatory radiation side effects, and treated with topical steroids and antibiotics. Despite the treatment, the anterior chamber reaction increased, showing more cells and a new hypopyon. A neurotrophic corneal ulcer also appeared, with a perforation occluded by the anterior chamber content, findings that were interpreted as pseudouveitis/pseudohypopyon due to intraocular recurrence of carcinoma and neurotrophic ulcer with corneal perforation oc- 
Fig. 2. Histopathological and immunohistochemical findings are shown. a Light microscopy showing a ciliar tumor in the nasal side, invading the sclera up to the subconjunctival space. HE. $1 \times$. The temporal side exhibits ciliary body fibrosis and scarring without signs of recurrence. b Immunohistochemical stain with CKAE1-AE3 $4 \times$ showing infiltration by lobules and nests of well-differentiated squamous carcinoma coming from the supraciliar space reaching the limbus, angle, and cornea. c Central cornea exhibiting a large central ulcer with perforation without inflammation and infiltrating lobules of squamous cell carcinoma in the anterior chamber and adhering to the posterior area. HE. $10 \times$. d Immunohistochemical stain with CKAE1-AE3 $10 \times$ corresponds to the same area with different staining in order to better visualize the tumor invasion.
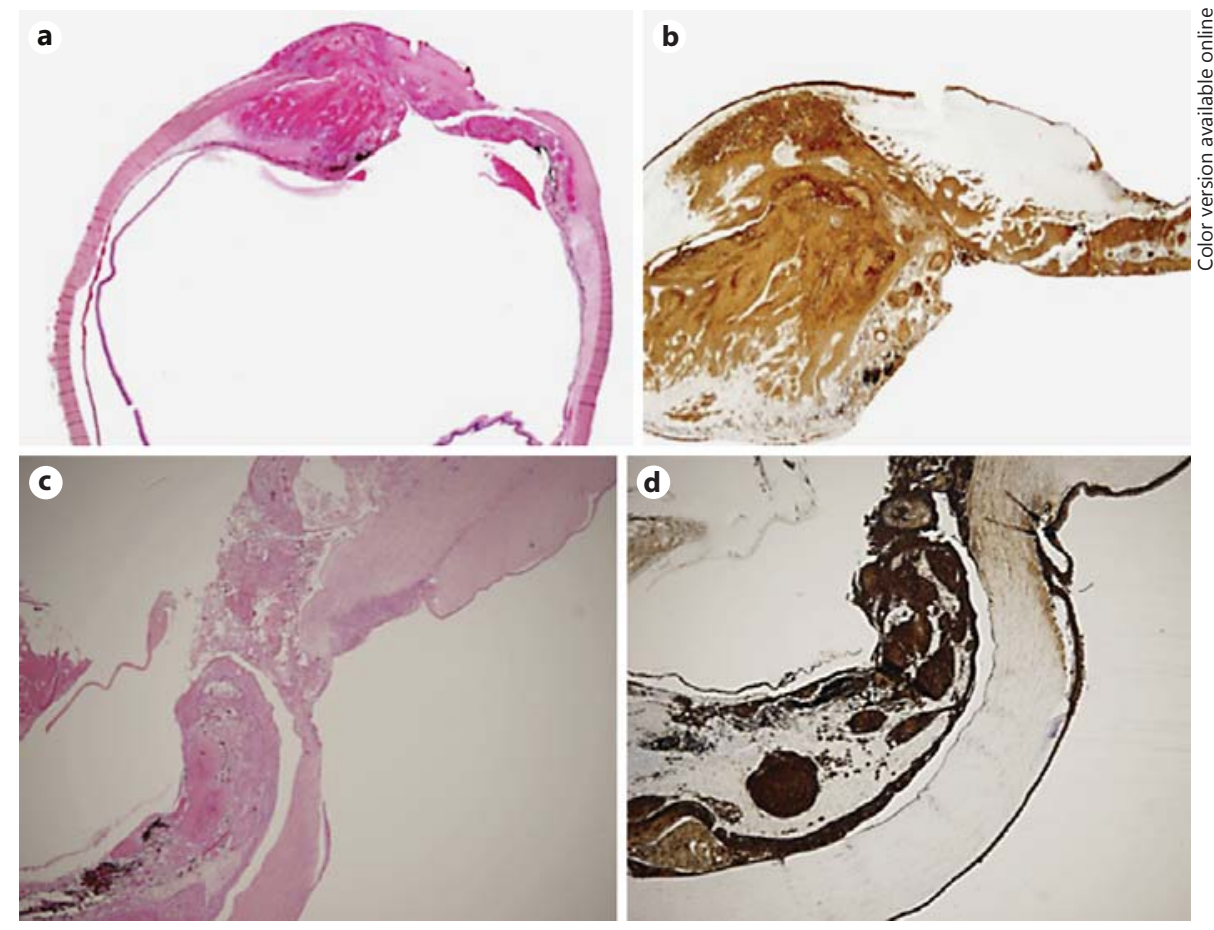

cluded by carcinoma cells (Fig. 1c). Magnetic resonance imaging showed an intraocular mass involving the nasal region without any signs of orbital involvement (Fig. 1d). No conjunctival recurrence was noted. Oncologic examination was negative for regional or systemic extension. The CSCC was suspected to have invaded intraocularly and caused a corneal perforation. Therefore, the left eye was enucleated. Histopathologic findings showed temporal scarring of the ciliary body with fibrosis, atrophy, and disorganization of the ciliary epithelium involving the peripheral choroid. These changes were consistent with the side effects of radiation therapy. Nasally, the conjunctiva showed no signs of local recurrence. However, the sclera displayed scarring and melting with a lobular infiltration of well-differentiated squamous cell carcinoma, originating from the supraciliar space and reaching the limbus, angle, and cornea (Fig. 2a, b). The central cornea exhibited a large ulcer with perforation without inflammation and additional infiltrating lobules of squamous cell carcinoma adhering to the posterior area (Fig. 2c, d). The final diagnosis was intraocular infiltrative recurrence of CSCC (without conjunctival recurrence) after resection and adjuvant brachytherapy.

\section{Discussion}

A review of the 360 cases of conjunctival tumors diagnosed at the Ocular Oncology Unit of the Valladolid University Hospital revealed that 124 (39.6\%) were of epithelial origin and only 11 were malignant CSCC [4]. This is the first case of recurrence and intraocular invasion treat- ed with enucleation of the eye. The management of CSCC in ocular oncology includes incisional/excisional biopsy with margins and adjuvant therapies with antimetabolites, such as mitomycin C or interferon drops, used postoperatively in order to treat the residual tumor or margins and to prevent local recurrences. When scleral invasion is suspected, brachytherapy is the next step in treatment. In the present case, our patient refused the topical treatment and only brachytherapy was opted for.

Intraocular invasion is rare and has been reported in $2-8 \%$ of cases $[1,5]$. It may arise along drainage routes or nerves, in addition to tracts created by previous trauma or intraocular surgery. In our case, the corneal incision for cataract extraction incision may have been the tract for intraocular invasion. Signs suggestive of intraocular invasion include scleritis, anterior synechiae, anterior chamber cells/pseudouveitis, contiguous intraocular mass, or spontaneous perforation of the globe. Careful clinical examination and ultrasound biomicroscopy are very useful for detecting early intraocular invasion [6, 7].

Brachytherapy and other forms of external radiation have been used for many years in the treatment of OSSN. Complications include radiation conjunctivitis, dry eye, cataract, scarring, symblepharon, ulceration, and corneal perforation $[5,8]$. However, no clinicopathologic reports 
of the tissue effects of radiation were found in the literature.

Histopathologic effects of brachytherapy and proton beam irradiation in uveal melanomas have been previously reported. These include necrosis, fibrosis, and blood vessel damage [9]. Conversely, no studies have reported these effects in anterior structures of the eye for OSSN. Our finding of a complete radiation-induced fibrous scar anteriorly is the first of its kind in the literature. In addition, a neurotrophic corneal ulcer developed secondary to radiation treatment and eventually perforated, presenting with similar histopathologic characteristics as in any type of radiation [10].

In the present case, the extensive scarring in the temporal area demonstrated the efficacy of the treatment. The neurotrophic corneal ulcer and perforation can be attributed to limbal insufficiency caused by a combination of surgery and radiation treatment. The intraocular diffuse recurrence suggests that a subclinical infiltration of carcinoma cells through the suprachoroidal space was already present at the time of the adjuvant treatment. These cells possibly escaped the adjuvant brachytherapy, extending up to the anterior chamber, masquerading as a pseudouveitis and invading the sclera from inside the globe up to its surface. The most likely route of early intraocular invasion was the surgical wound from previous cataract surgery.

In patients presenting with CSCC with a history of previous trauma or intraocular surgery, careful clinical ex- amination should be performed for early identification of intraocular invasion. To our knowledge, this is the first documented case of intraocular diffuse recurrence of CSCC after resection and adjuvant brachytherapy, without conjunctival relapse and with clinicopathologic correlation of radiation effects on the ocular tissues.

\section{Acknowledgements}

The authors are grateful to Jóse L. Sarasa Corral, MD, PhD, Pathology Department, Fundación Jiménez Díaz, Madrid, España, and to Cyril Archambault, McGill University, Montréal, QC, Canada.

\section{Statement of Ethics}

Informed consent was obtained from the patient for publication of this study.

\section{Disclosure Statement}

None of the authors has any potential conflicts of interest to disclose.

\section{Funding Sources}

No specific financial support was available for this study.

\section{References}

1 Kiire CA, Srinivasan S, Karp CL: Ocular Surface Squamous Neoplasia. Int Ophthalmol Clin 2010;50:35-46.

2 Alves LF, Fernandes BF, Burnier JV, Zoroquian P, Eskenazi DT, Burnier MN: Incidence of epithelial lesions of the conjunctiva in a review os 12,102 specimens in Canada. Arq Bras Oftalmol 2011;74:21-23.

3 Emmanuel B, Ruder E, Lin SW, Abnet C, Hollenbeck A, Mbulaiteye S: Incidence of squamous-cell carcinoma of the conjunctiva and other eye cancers in the NIH-AARP Diet and Health Study. Ecancer 2012;6:254.

4 Saornil MA, Becerra E, Mendez MC, Blanco G: Conjunctival tumors. Arch Soc Esp Oftal 2009;84:7-22.
5 Shields CL, Demirci H, Karatza E, Shields JA: Clinical survey of 1,643 melanocytic and non melanocytic conjunctival tumors. Ophthalmology 2004;11:1747-1754.

6 Zang Z, Li B, Xu X, Li L, Gao F: intraocular extension of conjunctival squamous cell carcinoma. Ophthalmologica 2007;221:200-203.

7 Rootman DB, McGovwan HD, Yucel YH, Pavlin CJ, Simpson ER: Intraocular extension of conjunctival invasive squamous cell carcinoma after pterygium surgery and cataract extraction. Eye Contact Lens 2012;38:133136.
8 Walsh-Conway N, Conway RM: Plaque brachytherapy for the management of ocular surface malignancies with corneoscleral invasion. Clin Exp Ophthalmol 2009;37:577-583.

9 Saornil MA, Fisher MR, Campbell RJ, Robertson DM, Earle JD, Eagle RC Jr, Shields JA, Shields CL, Chandra SR, Albert DM: Histopathologic study of eyes after iodine 125 episcleral plaque irradiation for uveal melanoma. Arch Ophthalmol 1997;115:1395-1400.

10 Chaqués V, Saornil MA, Herreras JM: Corneal perforation due to late radiation therapyinduced corneal necrosis. Clinicopathological correlation. Ocul Immunol Inflam 2000;8: $67-71$. 